\title{
ROBUST TRACKING CONTROL OF A QUADROTOR HELICOPTER WITHOUT VELOCITY MEASUREMENT
}

\author{
STEVANOVIC, S[tojan]; KASAC, J[osip] \& STEPANIC, J[osip]
}

\begin{abstract}
In this paper, a robust output tracking controller for quadrotor helicopter is proposed. The proposed controller requires measurement of only four state variables: positions in inertial coordinate frame and yaw angle. Also, the controller is robust to unmodeled dynamics and provides rejections of all external force and torque disturbances. The effectiveness of the proposed controller is tested on a simulation example of quadrotor tracking under wind influence which is modeled as unmatched external force disturbances in horizontal plane.

Keywords: Quadrotor helicopter, tracking control, robust control, output control
\end{abstract}

\section{INTRODUCTION}

The quadrotor helicopter is a small agile vehicle controlled by four rotors. Compared with other flying vehicles, quadrotors have specific characteristics that allow execution of applications that would be difficult or impossible otherwise. This superiority is due to their unique ability for vertical, stationary and low speed flight. The quadrotor also has higher payload capacity compared to conventional flying vehicles. The main disadvantage of quadrotor is its high energy requirement because it uses four motors.

From the control point of view, quadrotor is a highly nonlinear, multivariable, strongly coupled and underactuated system which has six degrees of freedom and only four actuators. The low cost and simplicity of mechanical structure mean the quadrotor provides an excellent testing ground for application of advanced control techniques.

Various advanced control methods have been developed, such as feedback linearization method [1, 2], adaptive control [3, 4], sliding-mode control [5], backstepping control [6], $\mathrm{H}_{\infty}$ robust control [7], etc. However, the most of proposed method require full information on the state that may limit their practical applicability, because the increased number of sensors makes the overall system more complex in implementation and expensive in realization.

With the aim of reducing the number of sensors, several solutions were proposed. In [8], the authors proposed a velocities estimator for a tracking control of an under-actuated quadrotor UAV using only linear and angular positions. In [9] and [10], sliding-mode observers were proposed to estimate the effect of external perturbations using measurement of positions and yaw angle.

The main practical difficulties of quadrotor tracking control are parametric uncertainties, unmodeled dynamics, and external disturbances. During the quadrotor flights, sudden wind-gusts can significantly affect flight performance and even cause instability [11, 12]. In order to accomplish high level tracking performances, robust flight control systems are required to track desired trajectories in the presence of wind or other disturbances. Recently, several solutions were proposed for reducing influence of wind disturbances on quadrotor tracking performances, mostly based on sliding-mode disturbance observers [13, 14]. The proposed control laws are based on full-state measurement, which require large number of sensors. Further, the outer feedback loop is based on feedback linearization of full translational dynamics of quadrotor, leading to computationally expensive control laws.

In this paper we propose a robust output tracking controller, which requires measurement of quadrotor linear position and yaw angle only. The proposed controller provides rejection of external force and torque disturbances like wind gusts. The control design is based on a bilinear reduced model of quadrotor, which preserves the relative degree of the system. The resulting outer control-loop, which is based on feedback linearization, has very simple structure compared to controllers described in literature. The inner control-loop is based on sliding-mode control design with the aim of compensating all disturbances and unmodeled dynamics. Further, sliding-mode filters are used to estimates all derivatives in control law providing significant reduction of required sensors. Also, a smoothing nonlinear filter is used to prevent sudden jumps of control variables.

The paper is organized as follows. In Section 2, the dynamic model of the quadrotor helicopter is developed. Based on this nonlinear dynamic model, in Section 3 is designed a robust output tracking controller. The simulation results are presented in Section 4. Finally, the concluding remarks are emphasized in Section 5.

\section{QUADROTOR DYNAMIC MODEL}

The quadrotor helicopter is made of a rigid cross frame equipped with four rotors. The equations describing the altitude and the attitude motions of a quadrotor helicopter are basically same as those describing a rotating rigid body with six degrees of freedom $[15,16]$.

\subsection{Quadrotor attitude dynamics and kinematics}

The three-axis rotational dynamic of rigid body in body-fixed reference frame is given by

$$
\mathbf{I} \dot{\boldsymbol{\omega}}+\boldsymbol{\omega} \times(\mathbf{I} \boldsymbol{\omega})=\boldsymbol{\tau}+\mathbf{d}_{\tau},
$$


where $\mathbf{I}=\operatorname{diag}\left\{I_{x}, I_{y}, I_{z}\right\}$ is the diagonal inertia matrix, $\boldsymbol{\omega}=\left[\begin{array}{lll}p & q & r\end{array}\right]^{\mathrm{T}}$ is the angular velocity vector, $\boldsymbol{\tau}=\left[\begin{array}{ll}\tau_{\phi} \tau_{\theta} \tau_{\psi}\end{array}\right]^{\mathrm{T}}$ is the vector of actuator torques, and $\mathbf{d}_{\tau}=\left[\begin{array}{lll}d_{\phi} & d_{\theta} & d_{\psi}\end{array}\right]^{\mathrm{T}}$ is the vector of external disturbance torques.

The rigid body rotational kinematics equations are given by

$$
\dot{\boldsymbol{\eta}}=\boldsymbol{\Omega}_{B} \boldsymbol{\omega}
$$

where $\boldsymbol{\eta}=[\phi \theta \psi]^{\mathrm{T}}$ are Euler angles defined according to the $x y z$-convention, and is the transformation matrix from body to inertial coordinate frame, where $c_{\eta} \equiv \cos (\eta)$ and $s_{\eta} \equiv \sin (\eta)$.

$$
\boldsymbol{\Omega}_{B}=\frac{1}{c_{\theta}}\left[\begin{array}{ccc}
c_{\theta} & s_{\phi} s_{\theta} & c_{\phi} s_{\theta} \\
0 & c_{\phi} c_{\theta} & -s_{\phi} c_{\theta} \\
0 & s_{\phi} & c_{\phi}
\end{array}\right],
$$

2.2 Quadrotor translational dynamics and kinematics

The translational dynamic model of rigid body in the body-fixed reference frame is given by

$$
m \dot{\mathbf{v}}+\boldsymbol{\omega} \times(m \mathbf{v})=\mathbf{F}+\mathbf{d}_{F},
$$

where $m$ is the mass of quadrotor, $\mathbf{F}=\left[F_{x} F_{y} F_{z}\right]^{\mathrm{T}}$ is the

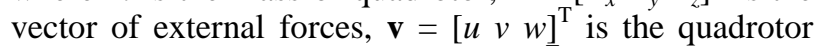
linear velocity, and $\mathbf{d}_{F}=\left[\begin{array}{lll}d_{x} & d_{y} & d_{z}\end{array}\right]^{\mathrm{T}}$ is the vector of external disturbance forces.

The rigid body translational kinematics equations are given by

$$
\dot{\xi}=\mathbf{R v}
$$

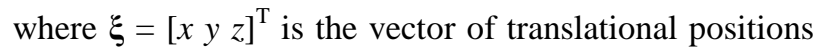
in inertial coordinate frame, and $\mathrm{R}$ is the rotation matrix given by

$$
\mathbf{R}=\mathbf{R}_{z}^{T}(\psi) \mathbf{R}_{y}^{T}(\theta) \mathbf{R}_{x}^{T}(\phi),
$$

where particular rotational matrices are

$$
\begin{gathered}
\mathbf{R}_{x}(\phi)=\left[\begin{array}{ccc}
1 & 0 & 0 \\
0 & c_{\phi} & s_{\phi} \\
0 & -s_{\phi} & c_{\phi}
\end{array}\right], \quad \mathbf{R}_{y}(\theta)=\left[\begin{array}{ccc}
c_{\theta} & 0 & s_{\theta} \\
0 & 1 & 0 \\
-s_{\theta} & 0 & c_{\theta}
\end{array}\right],(7) \\
\mathbf{R}_{z}(\psi)=\left[\begin{array}{ccc}
c_{\psi} & s_{\psi} & 0 \\
-s_{\psi} & c_{\psi} & 0 \\
0 & 0 & 1
\end{array}\right] .
\end{gathered}
$$

\subsection{Quadrotor forces and moments}

The four propellers of quadrotor, rotating at angular velocities $\Omega_{i}$ produce four forces $f_{i}=k_{i} \Omega_{i}^{2}$, directed upward, where $i=1,2,3,4$, and $k_{i}$ are positive constants. The use of the four forces $f_{i}$ as input to the system is somewhat counterintuitive. Thus, it is common to use a control allocation scheme that transforms the forces $f_{i}$ into a vertical thrust $\mathbf{F}=\left[\begin{array}{lll}0 & 0 & F_{z}\end{array}\right]^{\mathrm{T}}$ and three torques around the three orthogonal body axes $\tau_{\phi}, \tau_{\theta}, \tau_{\psi}$.

Thus, the control distribution from the four actuator motors of the quadrotor is given by

$$
\left[\begin{array}{l}
F_{z} \\
\tau_{\phi} \\
\tau_{\theta} \\
\tau_{\psi}
\end{array}\right]=\left[\begin{array}{cccc}
1 & 1 & 1 & 1 \\
-l & 0 & l & 0 \\
0 & l & 0 & -l \\
c & -c & c & -c
\end{array}\right]\left[\begin{array}{l}
f_{1} \\
f_{2} \\
f_{3} \\
f_{4}
\end{array}\right],
$$

where $l$ is the distance from the motors to the centre of gravity, and $c$ is a constant known as force-to-moment scaling factor. So, if required thrust and torque vector are given then the rotor force can be calculated using (9).

\subsection{Simplified dynamic model of quadrotor}

During the quadrotor navigation with moderate velocity, the roll and pitch angles remain near zero degrees to allow approximation of matrix $\boldsymbol{\Omega}_{B}$ with identity matrix, thus vector of derivation of the Euler angles $\dot{\boldsymbol{\eta}}$ can be approximated by the body axis angular velocity $\omega$. Under these assumptions, dynamic model of quadrotor (1)-(8) can be reduced to more appropriate form for control system design

$$
\begin{aligned}
& m \ddot{x}=\left(c_{\psi} s_{\theta} c_{\phi}+s_{\psi} s_{\phi}\right) u_{1}+d_{x} \\
& m \ddot{y}=\left(s_{\psi} s_{\theta} c_{\phi}-c_{\psi} s_{\phi}\right) u_{1}+d_{y}, \\
& m \ddot{z}=-m g+\left(c_{\phi} c_{\theta}\right) u_{1}+d_{z} \\
& I_{x} \ddot{\phi}=\left(I_{y}-I_{z}\right) \dot{\theta} \dot{\psi}+u_{2}+d_{\phi} \\
& I_{y} \ddot{\theta}=\left(I_{z}-I_{x}\right) \dot{\phi} \dot{\psi}+u_{3}+d_{\theta}, \\
& I_{z} \ddot{\psi}=\left(I_{x}-I_{y}\right) \dot{\phi} \dot{\theta}+u_{4}+d_{\psi}
\end{aligned}
$$

where

$$
u_{1}=F_{z}, \quad u_{2}=\tau_{\phi}, \quad u_{3}=\tau_{\theta}, \quad u_{4}=\tau_{\psi},
$$

are control variables, and $d_{x}, d_{y}, d_{z}, d_{\phi}, d_{\theta}, d_{\psi}$ are external disturbances.

\section{OUTPUT TRACKING CONTROL}

The quadrotor is an under-actuated system, because six state variables $\{x, y, z, \phi, \theta, \psi\}$ should be controlled by only four inputs $\left\{F_{z}, \tau_{\phi}, \tau_{\theta}, \tau_{\psi}\right\}$. Therefore, in most cases, only some of the system variables, for example $\{x$, $y, z, \psi\}$, are usually controlled.

The proposed robust output controller synthesis has three main phases: a) feedback linearization; b) slidingmode derivative filters design; c) sliding-mode control design.

\subsection{Feedback linearization controller (FLC) design}

The feedback linearization of control design on model (10), (11) leads to extremely complicated control law. But, if the feedback linearization is just first step of robust sliding-mode controller design, we can use additional simplification of model (10), (11) which preserve relative degree of the system. Under assumption of small angles $\eta_{i}, i=1,2,3$, follows that $\cos \left(\eta_{i}\right) \approx 1$ and $\sin \left(\eta_{i}\right) \approx \eta_{i}$. Further, neglecting the quadratic terms, the model (10), (11) becomes

$$
\begin{aligned}
& m \ddot{x}=\theta u_{1}+d_{m x} \\
& m \ddot{y}=-\phi u_{1}+d_{m y}, \\
& m \ddot{z}=-m g+u_{1}+d_{m z}
\end{aligned}
$$




$$
\begin{aligned}
& I_{x} \ddot{\phi}=u_{2}+d_{m \phi} \\
& I_{y} \ddot{\theta}=u_{3}+d_{m \theta}, \\
& I_{z} \ddot{\psi}=u_{4}+d_{m \psi}
\end{aligned}
$$

which is a bilinear system with the same relative degrees as the system (10), (11). Disturbances $d_{m x}, d_{m y}, d_{m z}, d_{m \phi}$, $d_{m \theta}, d_{m \psi}$ represent external disturbances and modelling errors due to simplification of original system (10), (11).

The control goal is tracking of desired quadrotor position $\left\{x_{d}(t), y_{d}(t), z_{d}(t)\right\}$, preserving angle $\psi_{d}=0$, in presence of unknown external disturbances.

So, the tracking error variables are

$$
\tilde{x}=x-x_{d}(t), \quad \tilde{y}=y-y_{d}(t), \quad \tilde{z}=z-z_{d}(t) .
$$

The first step is design of control law for $u_{1}$ which will stabilize the altitude tracking error

$$
m \ddot{\tilde{z}}=-m \ddot{z}_{d}-m g+u_{1}+d_{m z},
$$

The choice of control variable leads to error dynamics

$$
\begin{aligned}
& u_{1}=m\left(\ddot{z}_{d}+g-k_{z 1} \dot{\tilde{z}}-k_{z 0} \tilde{z}\right)+u_{1 s}, \\
& \ddot{\tilde{z}}+k_{z 1} \dot{\tilde{z}}+k_{z 0} \tilde{z}=\frac{1}{m}\left(u_{1 s}+d_{m z}\right),
\end{aligned}
$$

where $k_{z 1}$ and $k_{z 0}$ are positive gains, and $u_{1 s}$ is slidingmode part of controller which will be designed to compensate unknown disturbance $d_{m z}$. In that case, right side of eq. (18) converges to zero and also altitude error converges to zero for $k_{z 1}, k_{z 0}>0$.

The tracking error $\tilde{x}$ will be stabilized through the control variable $u_{3}$, since the first equation in (13) depends only on angle $\theta$. The second order error equation is

$$
m \ddot{\tilde{x}}=-m \ddot{x}_{d}+\theta u_{1}+d_{m x} .
$$

The equation (19) should be derived twice to provide explicit appearance of control variable $u_{3}$. So, the first derivation of (19) is

$$
m \dddot{\tilde{x}}=-m \dddot{x}_{d}+\dot{\theta} u_{1}+\theta \dot{u}_{1}+\dot{d}_{m x} .
$$

and the second derivation is

$$
m \tilde{x}^{(4)}=-m x_{d}^{(4)}+\ddot{\theta} u_{1}+2 \dot{\theta} \dot{u}_{1}+\theta \ddot{u}_{1}+\ddot{d}_{m x} .
$$

Inserting second equation of (14) in (21), we get

$$
m \tilde{x}^{(4)}=-m x_{d}^{(4)}+u_{1} \frac{u_{3}+d_{m \theta}}{I_{y}}+2 \dot{\theta}_{\dot{u}_{1}}+\theta \ddot{u}_{1}+\ddot{d}_{m x} .
$$

The choice of control variable

$$
u_{3}=\frac{I_{y}}{u_{1}}\left(m x_{d}^{(4)}-2 \dot{\theta} \dot{u}_{1}-\theta \ddot{u}_{1}+m u_{x}\right)+u_{3 s},
$$

where

$$
u_{x}=-k_{x 3} \dddot{\tilde{x}}-k_{x 2} \ddot{\tilde{x}}-k_{x 1} \dot{\tilde{x}}-k_{x 0} \tilde{x} .
$$

leads to error dynamics

$$
\tilde{x}^{(4)}+k_{x 3} \dddot{\tilde{x}}+k_{x 2} \ddot{\tilde{x}}+k_{x 1} \dot{\tilde{x}}+k_{x 0} \tilde{x}=\frac{u_{1}}{m I_{y}}\left(u_{3 s}+d_{c x}\right),
$$

where $k_{x 3}, k_{x 2}, k_{x 1}$ and $k_{x 0}$ are positive gains that satisfy Hurwitz stability condition, and $u_{3 s}$ is sliding-mode part of controller which will be designed to compensate cumulative disturbance $d_{c x}$

$$
d_{c x}=\frac{I_{y}}{u_{1}} \ddot{d}_{m x}+d_{m \theta} .
$$

The tracking error $\tilde{y}$ will be stabilized through the control variable $u_{2}$, since the second equation in (13) depends only on angle $\phi$. The second, third and fourth order error equation are

$$
\begin{gathered}
m \ddot{\tilde{y}}=-m \ddot{y}_{d}-\phi u_{1}+d_{m y} \\
m \ddot{\tilde{y}}=-m \dddot{y}_{d}-\dot{\phi} u_{1}-\phi \dot{u}_{1}+\dot{d}_{m y} \\
m \tilde{y}^{(4)}=-m y_{d}^{(4)}-u_{1} \frac{u_{2}+d_{m \phi}}{I_{x}}-2 \dot{\phi} \dot{u}_{1}-\phi \ddot{u}_{1}+\ddot{d}_{m y}
\end{gathered}
$$

The choice of control variable

$$
u_{2}=-\frac{I_{x}}{u_{1}}\left(m y_{d}^{(4)}-2 \dot{\phi} \dot{u}_{1}-\phi \ddot{u}_{1}+m u_{y}\right)-u_{2 s},
$$

where

$$
u_{y}=-k_{y 3} \dddot{\tilde{y}}-k_{y 2} \ddot{\tilde{y}}-k_{y 1} \dot{\tilde{y}}-k_{y 0} \tilde{y} .
$$

leads to error dynamics

$$
\tilde{y}^{(4)}+k_{y 3} \dddot{\tilde{y}}+k_{y 2} \ddot{\tilde{y}}+k_{y 1} \dot{\tilde{y}}+k_{y 0} \tilde{y}=\frac{u_{1}}{m I_{x}}\left(u_{2 s}+d_{c y}\right),
$$

where $k_{y 3}, k_{y 2}, k_{y 1}$ and $k_{y 0}$ are positive gains that satisfy Hurwitz stability conditions, and $u_{2 s}$ is sliding-mode part of controller which will be designed to compensate cumulative disturbance $d_{c y}$

$$
d_{c y}=\frac{I_{x}}{u_{1}} \ddot{d}_{m y}-d_{m \phi} .
$$

Finally, the angle $\psi$ can be stabilized by PD controller so that error dynamics has the following form

$$
\begin{gathered}
u_{4}=-I_{z} k_{\psi 1} \dot{\psi}-I_{z} k_{\psi 0} \psi, \\
\ddot{\psi}+k_{\psi 1} \dot{\psi}+k_{\psi 0} \psi=\frac{1}{I_{z}}\left(u_{4 s}+d_{m \psi}\right),
\end{gathered}
$$

which is asymptotically stable for $k_{\psi 1}, k_{\psi 0}>0$. Variable $u_{4 s}$ is sliding-mode part of controller which will be designed to compensate unknown disturbance $d_{m z}$.

It can be seen that overall feedback linearization controller (FLC) is a full-state controller, what means that require measurement of all 12 state variables. Also, FLC cannot be applied in the presence of unmatched external disturbances, since the second and third error derivatives (19), (20), (27), (28), which are necessary for controllers (24) and (31), contain unknown disturbances $d_{m x}, d_{m y}$, and their first derivations.

Note that the proposed control laws can guarantee convergent tracking only if control variable $u_{1}$ is strictly positive $u_{1}>0$, what can be achieved by including a saturation function in control law (17) 


$$
u_{1}=m g+k_{g} \tanh \left(\frac{m}{k_{g}}\left(\ddot{z}_{d}-k_{z 1} \dot{\tilde{z}}-k_{z 0} \tilde{z}\right)+\frac{1}{k_{g}} u_{1 s}\right),
$$

where positive parameter $k_{g}$ satisfies condition: $k_{g}<m g$.

\subsection{Sliding-mode controller (SMC) design}

The sliding-mode control laws are chosen to compensate external and unmodeled disturbances. The error dynamics (18) can be represented as

$$
\dot{s}_{1}+\lambda_{1} s_{1}=\frac{1}{m}\left(u_{1 s}+d_{m z}\right),
$$

where the sliding variable $s_{1}$ has the following form

$$
s_{1}=\dot{\tilde{z}}+\alpha_{z 0} \tilde{z} .
$$

Positive parameters $\lambda_{1}$ and $\alpha_{\mathrm{z} 0}$ satisfy the following conditions

$$
k_{z 1}=\alpha_{z 0}+\lambda_{1}, \quad k_{z 0}=\alpha_{z 0} \lambda_{1} .
$$

The sliding-mode controller $u_{1 s}$ will be chosen as

$$
u_{1 s}=-\rho_{1} \operatorname{sign}\left(s_{1}\right)=-\rho_{1} \frac{s_{1}}{\left|s_{1}\right|},
$$

where $\rho_{1}$ is a positive parameter which will be defined by using Lyapunov stability analysis. Taking the Lyapunov function we get time derivation where we used (37) and (40), so that

$$
\begin{gathered}
V_{1}=\frac{1}{2} s_{1}^{2}, \\
\dot{V}_{1}=s_{1} \dot{s}_{1}=s_{1}\left(-\lambda_{1} s_{1}+\frac{1}{m}\left(-\rho_{1} \frac{s_{1}}{\left|s_{1}\right|}+d_{m z}\right)\right), \\
\dot{V}_{1}=-\lambda_{1} s_{1}^{2}-\frac{\rho_{1}}{m}\left|s_{1}\right|+\frac{d_{m z}}{m} s_{1} \\
\leq-\lambda_{1} s_{1}^{2}-\frac{1}{m}\left(\rho_{1}-\max \left\{\left|d_{m z}\right|\right\}\right)\left|s_{1}\right|
\end{gathered}
$$

The time derivation of Lyapunov function will be negative definite if the following condition is satisfied

$$
\rho_{1}>\max \left\{\left|d_{m z}\right|\right\} .
$$

The similar approach can be applied to second-order error equation (35). The sliding-mode controller $u_{4 s}$ will be chosen as where sliding variable $s_{4}$ has the following form

$$
\begin{gathered}
u_{4 s}=-\rho_{4} \operatorname{sign}\left(s_{4}\right), \\
s_{4}=\dot{\psi}+\alpha_{\psi 0} \psi,
\end{gathered}
$$

so that error equation (35) becomes

$$
\dot{s}_{4}+\lambda_{4} s_{4}=\frac{1}{I_{z}}\left(u_{4 s}+d_{m \psi}\right),
$$

Positive parameters $\lambda_{4}, \alpha_{\psi 0}$ and $\rho_{4}$ satisfy the following conditions

$$
k_{\psi 1}=\alpha_{\psi 0}+\lambda_{4}, \quad k_{\psi 0}=\alpha_{\psi / 0} \lambda_{4}, \quad \rho_{4}>\max \left\{\left|d_{m \mu}\right|\right\} .
$$

The fourth-order error equation (25) can be represented as

$$
\dot{s}_{3}+\lambda_{3} s_{3}=\frac{u_{1}}{m I_{y}}\left(u_{3 s}+d_{c x}\right),
$$

where the sliding variable $s_{3}$ has the following form

$$
s_{3}=\dddot{\tilde{x}}+\alpha_{x 2} \ddot{\tilde{x}}+\alpha_{x 1} \dot{\tilde{x}}+\alpha_{x 0} \tilde{x} .
$$

Positive parameters $\lambda_{3}, \alpha_{x 2}, \alpha_{x 1}$ and $\alpha_{x 0}$ satisfy the following conditions

$$
\begin{array}{ll}
k_{x 3}=\alpha_{x 2}+\lambda_{3}, & k_{x 2}=\alpha_{x 1}+\lambda_{3} \alpha_{x 2}, \\
k_{x 1}=\alpha_{x 0}+\lambda_{3} \alpha_{x 1}, & k_{x 0}=\lambda_{3} \alpha_{x 0},
\end{array}
$$

including Hurwitz stability condition: $\alpha_{x 2} \alpha_{x 1}>\alpha_{x 0}$. The sliding-mode controller $u_{1 s}$ will be chosen as

$$
u_{3 s}=-\rho_{3} \operatorname{sign}\left(s_{3}\right),
$$

where the positive parameter $\rho_{3}$ satisfies the following condition

$$
\rho_{3}>\max \left\{\left|d_{c x}\right|\right\} .
$$

The similar sliding-mode control law can be derived for fourth-order error dynamic (32)

$$
\begin{aligned}
& u_{2 s}=-\rho_{2} \operatorname{sign}\left(s_{2}\right), \\
& s_{2}=\dddot{\tilde{y}}+\alpha_{y 2} \ddot{\tilde{y}}+\alpha_{y 1} \dot{\tilde{y}}+\alpha_{y 0} \tilde{y},
\end{aligned}
$$

where positive parameters $\rho_{2}, \alpha_{y 2}, \alpha_{y 1}$ and $\alpha_{y 0}$ satisfy similar conditions as (51) and (53).

The proposed sliding-mode controllers provide effective compensation of matched uncertainties $d_{m z}, d_{m \phi}$, $d_{m \theta}, d_{m \psi}$, but cannot provide compensation of unmatched uncertainties $d_{m x}, d_{m y}$, since the higher-order error derivatives contain unknown disturbances and their first derivatives. This problem can be avoided by introducing filters in the form of sliding-mode derivative estimators.

\subsection{Sliding-mode filter (SMF) design}

One of the approaches for estimation of time derivations of some continuous function $\zeta(t)$, is using sliding-mode filter (SMF)

$$
\begin{gathered}
\dot{\zeta}_{e}=v_{\zeta}=-\rho_{\zeta} \operatorname{sign}\left(\zeta_{e}-\zeta\right), \\
\rho_{\zeta}>\max (|\dot{\zeta}|) .
\end{gathered}
$$

where $\zeta_{e}$ is estimation of $\zeta$, and $v_{\zeta}$ is estimation of $\dot{\zeta}$. The gain $\rho_{\zeta}$ is tuned to satisfy the following condition. We use the SMF (55) to replace all time derivations in proposed control laws with their estimates. Input functions of SMF are and output derivation estimates are

$$
\begin{aligned}
& \zeta=\left[\begin{array}{llllllllll}
\tilde{x} & \tilde{y} & \tilde{z} & \dot{\tilde{x}}_{e} & \dot{\tilde{y}}_{e} & \ddot{\tilde{x}}_{e} & \ddot{\tilde{y}}_{e} & u_{1} & \dot{u}_{1, e} & \psi
\end{array}\right]^{T}, \\
& \dot{\zeta}_{e}=\left[\begin{array}{llllllllll}
\dot{\tilde{x}}_{e} & \dot{\tilde{y}}_{e} & \dot{\tilde{z}}_{e} & \ddot{\tilde{x}}_{e} & \ddot{\tilde{y}}_{e} & \ddot{\tilde{x}}_{e} & \dddot{\tilde{y}}_{e} & \dot{u}_{1, e} & \ddot{u}_{1, e} & \dot{\psi}_{e}
\end{array}\right]^{T} .
\end{aligned}
$$

On this way, we get output controller which requires measurement of only four output variables $\{x, y, z, \psi\}$, and provides also compensation of unmatched disturbances $d_{m x}, d_{m y}$.

Note that signum function which appears in slidingmode controllers and filters can cause chattering phenomena, or high-frequency oscillations of control 
variables. This problem can be avoided by replacing discontinuous signum function with appropriate continuous approximation, like

$$
\operatorname{sign}(\zeta) \approx \tanh (K \zeta)
$$

where positive scalar $K$ has some enough large value.

The initial conditions of the filters are set to zero, leading to large jumps of control variables at the beginning of control action. Usual approach to prevent this problem is filtration of desired reference trajectory through a linear six-order filter [9]. But, this filtration significantly deforms the initial reference trajectory. In this work, we use a saturated nonlinear smoothing filter, which preserves initial form of reference trajectory,

$$
\begin{gathered}
\dot{u}_{i, s}=-\rho_{u, i} \tanh \left(\mu_{i}\left(u_{i, s}-u_{i}\right)\right), \\
\rho_{u, i}>\max \left(\left|\dot{u}_{i}\right|\right)
\end{gathered}
$$

where $u_{i, s}$ is filtered form of the control variable $u_{i}$, and $\mu_{i}$ is a positive scalar. The gain $\rho_{u, i}$ is tuned to satisfy the following condition So, by gain $\rho_{u, i}$ we can reduce the maximal control slope.

\section{SIMULATION RESULTS}

In order to verify the effectiveness of the proposed control law, the overall system is tested in numerical simulations using Runge-Kutta's method with variable time step. The physical parameters for quadrotor are: $I_{x}=0.62 \mathrm{~N} \mathrm{~m} \mathrm{~s}^{2}, I_{y}=0.62 \mathrm{~N} \mathrm{~m} \mathrm{~s}^{2}, I_{z}=1.24 \mathrm{~N} \mathrm{~m} \mathrm{~s}^{2}$, $m=1 \mathrm{~kg}, g=9.81 \mathrm{~m} / \mathrm{s}^{2}$.

The reference trajectory chosen for $x_{d}(t), y_{d}(t), z_{d}(t)$ and $\psi_{\mathrm{d}}(t)$ is

$$
\begin{aligned}
& x_{d}(t)=\cos (0.5 t) \\
& y_{d}(t)=\sin (0.5 t) \\
& z_{d}(t)=0.5 t \\
& \psi_{d}(t)=0
\end{aligned}
$$

The initial conditions are: $x_{d}(0)=0.5 \mathrm{~m}, y_{d}(0)=0 \mathrm{~m}$, $z_{d}(0)=0 \mathrm{~m}$ and $\psi_{\mathrm{d}}(0)=0 \mathrm{rad}$. All other initial conditions are zero.

To test the robustness of the controller, disturbances have been introduced. The most likely disturbance acting on the quadrotor is wind in horizontal plane, which can be modeled by forces $d_{m x}, d_{m y}$, chosen as

$$
\begin{aligned}
& d_{m x}(t)=1.5+2.5 \sin (4 \mathrm{t}) \\
& d_{m y}(t)=2.5+1.5 \sin (3 \mathrm{t})
\end{aligned}
$$

All other external disturbances are set to zero.

The FLC+SMC controller gains for $\mathrm{u}_{1}$ and $\mathrm{u}_{4}$ are chosen as: $\lambda_{1}=3, \alpha_{\mathrm{z} 0}=3$ and $\rho_{1}=0$. The FLC + SMC controller gains for $\mathrm{u}_{2}$ and $\mathrm{u}_{3}$ are chosen as: $\lambda_{3}=3, \alpha_{x 2}=9$, $\alpha_{x 1}=27, \alpha_{x 0}=27$ and $\rho_{3}=4$. The SMF gains, which appear in (55) and (59) are $\rho_{\zeta}=40$ and $K=20$. The smoothing filter gains are $\rho_{u, i}=80$ and $\mu_{i}=5$.

In Figures 1-4 we can see simulation results for FLC+SMF in the case without external disturbances. We can see almost asymptotic convergence toward reference trajectory. The small steady-state error, which can be seen in Fig. 2, is a consequence of quadrotor model simplification. Such a small error and small values of roll and pitch angle, which can be seen in Fig. 1, justify starting assumptions for synthesis of FLC.

In Fig. 5 we can see simulation results for FLC combined with SMF in the case of external force disturbances. It can be seen that FLC with SMF cannot provide asymptotic tracking of reference trajectory. In Fig. 6 we can see simulation results for FLC combined with SMF and SMC in the case of external disturbances. It can be seen that sliding-mode part of the controller ensures efficient disturbance rejection, providing convergent tracking of reference trajectory.

In Fig 4. and Fig. 7. we can see forces and torques for FLC + SMF in the case without external disturbances, and for FLC + SMC + SMF in the case with external disturbances, respectively. In both cases there are no control jumps which appear in the case without smoothing filters (60).

Simulation results for other choices of reference trajectories and initial conditions show similar behavior. Also, controller shows high robustness to change in system parameters.

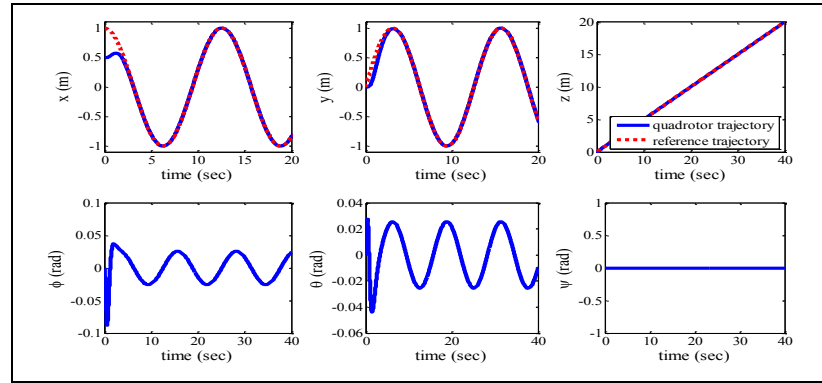

Fig. 1.The position and attitude of quadrotor in the closed-loop with FLC + SMF (the case without external disturbances)

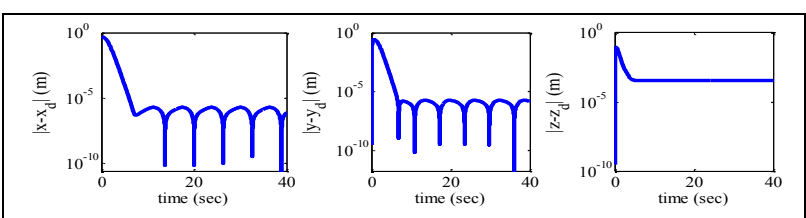

Fig. 2.The position errors of quadrotor in the closed-loop with FLC + SMF (the case without external disturbances)

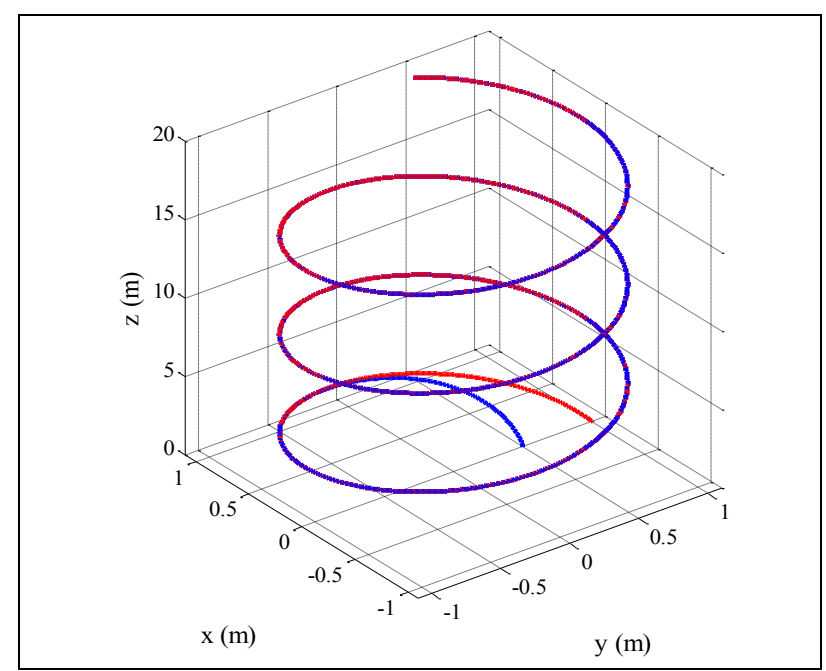

Fig. 3. The quadrotor and reference trajectory for case with FLC + SMF, without external disturbances 


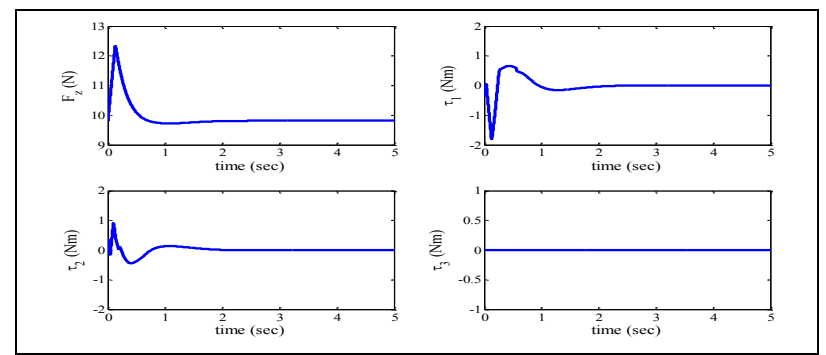

Fig. 4.The force and torques of quadrotor in the closed-loop with FLC + SMF (the case without external disturbances)

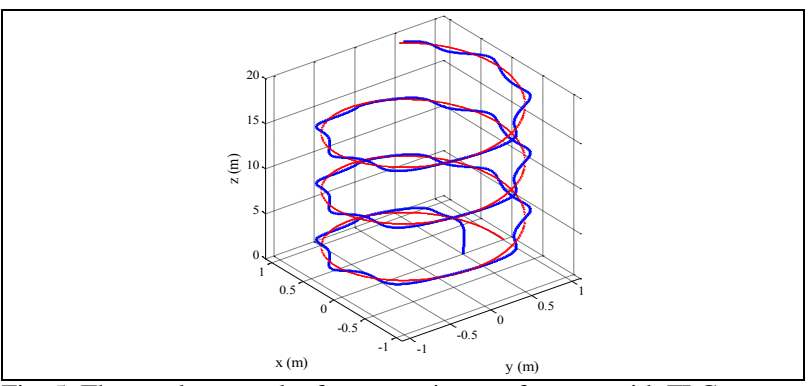

Fig. 5. The quadrotor and reference trajectory for case with FLC + SMF, with external disturbances

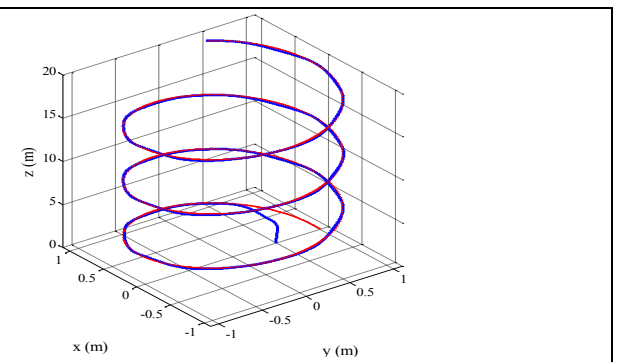

Fig. 6. The quadrotor and reference trajectory for case with FLC + SMC + SMF, with external disturbances

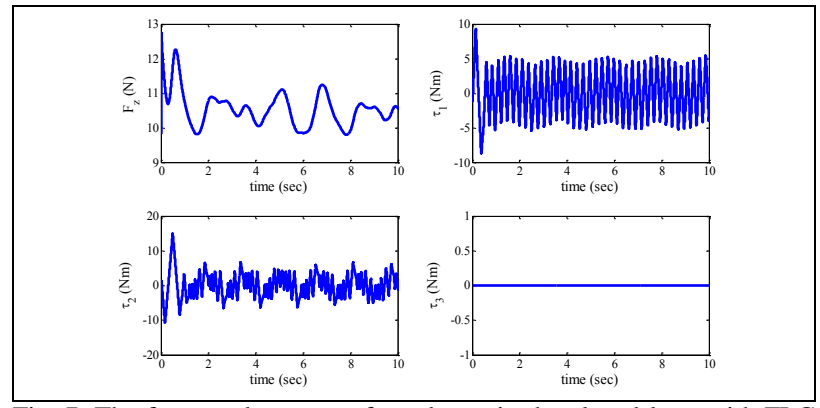

Fig. 7. The force and torques of quadrotor in the closed-loop with FLC $+\mathrm{SMC}+\mathrm{SMF}$, with external disturbances

\section{CONCLUSIONS}

In this paper, the performances of a robust output controller are demonstrated on a challenging problem: quadrotor tracking under unmatched external force disturbances in horizontal plane. The simulation results illustrate that proposed controller provides significant reduction of disturbances influence on quadrotor tracking performances. Such controller capability is important in real applications where wind influence cannot be avoided. The main drawback of the proposed controller is that there are no exact tuning rules for adjustment of filter gains.

The future work will be oriented toward exact Lyapunov-based stability analysis of the proposed controller with included sliding-mode filters. Such an analysis should provide explicit stability conditions for controller and filter gains depending on dynamic model parameters and amplitudes of external disturbances.

\section{ACKNOWLEDGEMENTS}

This work was supported by the National Scientific Foundation of the Republic of Croatia under Grant No. 120-1201842-3048. The authors would like to thank Teodor Tomic for helpful comments and discussions.

\section{REFERENCES}

[1] Voos, H. (2009). Nonlinear Control of a Quadrotor Micro-UAV using Feedback Linearization, Proceedings of the 2009 IEEE International Conference on Mechatronics, Malaga, Spain, April 14-17, ISBN 978-1-4244-4195-2

[2] Das, A.; Subbarao, K. \& Lewis F. (2009). Dynamic inversion with zero-dynamics stabilisation for quadrotor control, IET Control Theory and Applications, Vol. 3, pp. 303-314, ISSN 1751-8644

[3] Diao, C.; Xian, B.; Yin, Q.; Zeng, W.; Li, H. \& Yang, Y. (2011). A Nonlinear Adaptive Control Approach for Quadrotor UAVs, Proceedings of 2011 8th Asian Control Conference, Kaohsiung, Taiwan, May 15-18, ISBN 978-1-61284-487-9

[4] Nicol, C.; Macnab, C. J. B. \& Ramirez-Serrano, A. (2011). Robust adaptive control of a quadrotor helicopter, Mechatronics, vol. 21, pp. 927-938, ISSN 0957-4158

[5] Rong, X. \& Ozguner, U. (2006). Sliding Mode Control of a Quadrotor Helicopter, Proceedings of 45th IEEE Conference on Decision and Control, pp.4957-4962, San Diego, CA, USA, December 13-15, ISBN 1-4244-0171-2

[6] Madani, T. \& Benallegue, A. (2006). Control of a Quadrotor Mini-Helicopter via Full State Backstepping Technique, Proceedings of 45th IEEE Conference on Decision and Control, pp. 1515-1520, San Diego, CA, USA, December 13-15, ISBN 14244-0171-2

[7] Raffo, G. V.; Ortega, M. G. \& Rubio, F. R. (2010). An integral predictive/nonlinear $\mathrm{H}_{\infty}$ control structure for a quadrotor helicopter, Automatica, vol. 46, pp. 29-39, ISSN 0005-1098

[8] Lee, D. B.; Burg, T. C.; Xian, B. \& Dawson, D. M. (2007) Output feedback tracking control of an underactuated quad-rotor UAV, Proceedings of the 2007 American Control Conference, New York, USA, July 9-13, pp. 1775-1780, ISBN 1-4244-0989-6

[9] Madani, T. and Benallegue, A. (2007). Sliding mode observer and backstepping control for a quadrotor unmanned aerial vehicles, Proceedings of the 2007 American Control Conference, New York, USA, July 9-13, pp. 5887-5892, ISBN 1-4244-0989-6

[10] Benallegue, A.; Mokhtari, A. \& Fridman, L. (2008). High-order sliding-mode observer for a quadrotor UAV, International Journal of Robust and Nonlinear Control, vol. 18, pp. 427-440 ISSN 1099-1239

[11] Alexis, K.; Nikolakopoulos, G. \& Tzes, A. (2011). Switching Model Predictive Attitude Control for a Quadrotor Helicopter subject to Atmospheric Disturbances, Control Engineering Practice, vol. 19, pp. 1195-1207, ISSN 0967-0661

[12] Powers, C.; Mellinger, D.; Kushleyev, A.; Kothmann, B. \& Kumar, V. (2012). Influence of Aerodynamics and Proximity Effects in Quadrotor Flight, Proceedings of the 13th International Symposium on Experimental Robotics, Quebec City, Canada, June 17-21, ISBN 978-1-4577-0746-9

[13] Besnard, L.; Shtessel, Y. B. \& Landrum, B. (2012). Quadrotor vehicle control via sliding mode controller driven by sliding mode disturbance observer, Journal of the Franklin Institute, vol. 349 pp. 658-684, ISSN 0016-0032

[14] Escareno, J.; Salazar, S.; Romero, H. \& Lozano, R. (2012). Trajectory Control of a Quadrotor Subject to 2D Wind Disturbances, Journal of Intelligent \& Robotic Systems, Published Online: July 31, DOI 10.1007/s10846-012-9734-1, ISSN 09210296

[15] Fossen, T. I. (1994). Guidance and Control of Ocean Vehicles, John Wiley \& Sons, New York, ISBN 0-471-94113-1

[16] Castillo, P.; Lozano, R. \& Dzul, A. E. (2005). Modelling and control of mini flying machines, Springer-Verlag, London, ISBN 1-85233-957-8 